\title{
Further Report on Glyoxalase Content in Human Milk and Arakawa's Reaction.
}

108th Report of the Peroxidase Reaction.

By

Rokuro Orimo.

(折茂六郎)

(From the Department of Pediatrics, Faculty of Medicine, Tohoku Imperial University, Sendai.

Director: Prof. A. Sato.)

According to Dakin and Dudle ${ }^{12)(3)}$ there is, in practically all animal tissues except pancreds, an enzyme which converts glyoxals into corresponding hydroxyacids. They proposed to call it "Glyoxalase." As they state, the catalyst effects the rapid conversion of methyl glyoxal into lactic acid and of phenyl glyoxal into mandelic acid-a reaction which is readily imitated in vitro by the action of alkalies. The reaction is shown by the following formulas :-

$$
\begin{aligned}
& \mathrm{CH}_{3} \cdot \mathrm{CO} \cdot \mathrm{CHO}+\mathrm{H}_{2} \mathrm{O}=\mathrm{CH}_{3} \cdot \mathrm{CHOH} \cdot \mathrm{COOH} \\
& \mathrm{C}_{6} \mathrm{H}_{5} \cdot \mathrm{CO} \cdot \mathrm{CHO}+\mathrm{H}_{2} \mathrm{O}=\mathrm{C}_{6} \mathrm{H}_{5} \cdot \mathrm{CHOH} \cdot \mathrm{COOH} .
\end{aligned}
$$

They investigated also the effect of temperature and the influence of acid and alkali on glyoxalase. They also reported the presence of the enzyme in other organisms, in yeast and oysters, for instance.

About the same time, Neuber $\mathrm{g}^{4) 55}$ discovered the same enzyme in dog liver and muscle, and named it "Ketonaldehydmutase". In 1915 Dudle $y^{6}$ reported the quantitative method of glyoxalase in 5 c.c. of blood. In $1921 \mathrm{Findlay}^{7)}$ stated that the glyoxalase centent of the

1) H. D. Dakin and H. W. Dudley, J. of Biol. Chem., 1913, 14, 155.

2) H. D. D a kin and H. W. D ud ley, J. of Biol. Chem., 1913, 14, 423.

3) H. D. D a k in and H. W. Dudle y, J. of Biol. Chem., 1913, 15, 463.

4) C. N e u berg, Biochem. Zschr., 1913, 49, 502.

5) C. Ne uberg, Biochem. Zschr., 1913, 51, 484.

6) H. W. Dud le y, Biochem. J., 1915, 9, 255.

7) G. M. Fin d I a , Biochem. J., 1921, 15, 104. 
liver in pigeons with beriberi is less than that in control pigeons, and that an administration of vitamin- $\mathrm{B}^{*}$ to a beriberic pigeon is followed by an increase in the glyoxalase content of the liver. Fo s te $1^{8)}$ repeated Dakin and Dudley's experiment and obtained similar results with the exception that rabbit muscle appeared to contain no glyoxalase. Dudle $y^{9}$ showed the last observation to be erroneous by detecting the presence of the enzyme in weak alkalic $\left(\mathrm{Na}_{2} \mathrm{CO}_{3}\right)$ extract of rabbit muscle.

In 1928 Ariyama ${ }^{10)}$ repeated Dakin and Dudley's experiment and obtained similar results and stated that the optimum hydrogen ion concentration for the activity of glyoxalase lies in the neighbourhood of $\mathrm{pH} \mathrm{7,} \mathrm{and} \mathrm{that} \mathrm{the} \mathrm{glyoxalase} \mathrm{content} \mathrm{of} \mathrm{liver} \mathrm{is} \mathrm{higher} \mathrm{than} \mathrm{that} \mathrm{of}$ other animal tissues. In $1931 \mathrm{Vog} t-\mathrm{M} \phi \mid l \mathrm{er}^{11}$ found the decrease of liver enzyme in beriberic mice, and hypothesized that the symptoms of B-avitaminosis would be in a great part due to the intoxication of methyl glyoxal. In 1932 Ariy a ma ${ }^{12)}$ reported that the glyoxalase content of beriberic pigeons is less than that of hungry pigeons, though in both cases the glyoxalase content decreased, as he reported, compared with that in normal pigeons.

A. Takamatsu ${ }^{13)}$ in our Laboratory was able to isolate the methyl glyoxal-like substance from human milk negative to A rakawa's reaction.** He then supposed that the methyl glyoxal-like substance contained in such a milk would have a close connection with the glyoxalase. $\mathrm{He}^{14)}$ tried to estimate glyoxalase in human milk, and found that human milk positive to Arakawa's reaction contains much glyoxalase, and that Arakawa-negative milk contains only a small amount of it.

* Vitamin- $B_{1}$ is meant throughout the paper.

8) D. L. Foster, Biochem. J., 1925, 19, 757.

9) H. W. Dudley, Biochem. J., 1926, 20, 314.

10) N. A riy a m a, J. of Biol. Chem., 1928, 77, 359.

11) P. Vogt-M $\phi 1$ le r, Biochem. Zschr., 1931, 233, 248.

12) N. A riya ma, Nippon Seikagakkai Kaiho, 1932, 7, 168.

13) A. Takamatsu, Tohokn J. Exp. Med., 1935, 25, 575.

** Arakawa-positive may be used in two different senses. One of these is : Arakawa-positive in a biochemical sense. A sample of human milk is said to have become Arakawa-positive, when it becomes blue on the addition of Arakawa's reagent. Here it means that the sample is not negative to Arakawa's reaction. The other of these two senses is: Arakawa-positive in a clinical sense. A sample of human milk is clinically Arakawa-positive only when it shows such a reaction as $H$ or $\#$ in one minute of the addition of A rakawa's reagent. 'Another sample of human milk may be Arakawa-positive in the first described sense, but yet clinically negative.

14) A. Taka matsu, Tohoku J. Exp. Med, 1988, 34, 509. 


\section{Method of Experiment.}

Material milks were examined with Arakawa's reagent in each case and divided into three groups according to the intensity of Arakawa's reaction*; the Arakawa-positive, the Arakawa-weakly positive group, and the Arakawa-negative group. Glyoxalase was estimated by means of Dudley's method, as Takamats ${ }^{14)}$ described.

In each case milk was used 5 c.c.. 5 c.c. of $0.5 \%$ solution of phenyl glyoxal were put into a conical flask containing 5 c.c. of water and a few c.c. of a suspension of chalk** in water were added. A sample of milk to be examined was then added and mixed well. To this mixture a few drops of toluene were added and incubated at $37^{\circ}$ for 24 hours. After cooling, 2 c.c. of phosphoric acid were added and then extracted four times with a small quantity of ether (about 10 15 c.c.). Each ether layer was washed slowly with distilled water (about 5c.c.). The mandelic acid was completely removed by this process, the ether was then allowed to stand for about 20 hours mixed with small quantities of sodium sulphate anhydrous. This anhydrous ether was then removed by distillation and a residue of mandelic acid was obtained. Mandelic acid was then dissolved in 15 c.c. of distilled water. A pinch of animal charcoal was added to the hot solution, which was first allowed to cool and then filtered. The small amount of fat which usually appears in the ether residue was completely removed by this means. Then the solution was titrated with $\mathrm{N} / 100 \mathrm{NaOH}$ solution. And the value of titration indicated the content of glyoxalase. I measured the glyoxalase in 212 mothers or in 332 breasts in all.

\section{Result of Experiment.}

As above mentioned, all the cases were allocated into 3 groups according to the intensity of Arakawa's reaction. The result in the Arakawa-positive group in shown in Table 1, that in the weakly Arakawa-positive group in Table 2, and that in the Arakawa-negative group in Table 3 . In Table $4 \mathrm{I}$ estimated the content of glyoxalase in 23 cases with different Arakawa's reaction on both breasts, namely with Arakawa's reaction $5^{\prime}(-)$ on one breast.

** I used the chalk "De Luxe" made in U. S. A. 
As has been shown in Table 1 there were 16 cases with more than 1.0 of glyoxalase out of 59 cases $(27.1 \%)$ and the average content of glyoxalase was 0.73 in the Arakawa-positive group. In the weakly Arakawa-positive group, there were, as has been shown in Table 2, 30 cases with more than 1.0 out of 179 cases (16.8\%), and the average content was 0.52 . In the completely Arakawa-negative group (Cf. Table 3) there were 9 cases with more than 1.0 out of 94 cases $(9.6 \%)$ and the average content was 0.42 . In total there were 55 cases with more than 1.0 out of 332 cases $(16.7 \%)$ and the average content was 0.53 (Cf. Table 5).

If the cases were divided into right and left side breasts (Cf. Table 6), there were 29 right side breasts and 30 left side breasts in the Arakawa-positive group, and in the weakly Arakawa-positive group there were 99 cases of the right side breast and 80 cases of the left, and in the completely Arakawa-negative group there were 49 cases of the right and 45 cases of the left. As to the average content of glyoxalase, it was in the Arakawa-positive group 0.72 on the right side, 0.74 on the

TABLE 1.

Glyoxalase content in the Arakawa-positive group.

\begin{tabular}{|c|c|c|c|c|c|c|c|c|c|}
\hline \multirow{2}{*}{$\begin{array}{l}\text { Case } \\
\text { No. }\end{array}$} & \multirow{2}{*}{ Name } & \multirow{2}{*}{$\begin{array}{c}\text { Years } \\
\text { of } \\
\text { age }\end{array}$} & \multirow{2}{*}{$\begin{array}{l}\text { Month } \\
\text { of } \\
\text { experi- } \\
\text { ment }\end{array}$} & \multirow{2}{*}{$\begin{array}{l}\text { Diagnosis? } \\
\text { of the } \\
\text { infants }\end{array}$} & \multicolumn{2}{|c|}{$\begin{array}{l}\text { Arakawa's } \\
\text { reaction }\end{array}$} & \multicolumn{2}{|c|}{$\begin{array}{c}\text { Glyoxalase } \\
\text { content }\end{array}$} & \multirow{2}{*}{ 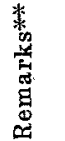 } \\
\hline & & & & & Right & Left & Right & Left & \\
\hline 1 & M. S. & 27 & IV & At & $1(\#)$ & $1(\#)$ & 0.3 & 1.2 & $*$ \\
\hline 2 & K. U. & 30 & 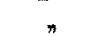 & G. I & $1(+)$ & $1(+t)$ & 0.5 & 0.5 & $\because \mathrm{Be}$ \\
\hline 3 & H. M. & 30 & 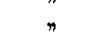 & $\mathrm{He}$ & $1(H)$ & $1(H)$ & 1.65 & 1.0 & \\
\hline 4 & Y. H. & 24 & & $\mathbf{E}$ & $1(H)$ & $1(H)$ & 0.6 & 0.4 & $\%$ \\
\hline 5 & M. I. & 22 & $\mathrm{~V}$ & D & $1(H)$ & $1(H)$ & 0.8 & 0.7 & \\
\hline 6 & Y. B. & 29 & 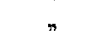 & D & $1(\mathrm{H})$ & $1(\mathrm{H})$ & 2.0 & & \\
\hline 7 & N.S. & 27 & $n$ & Dyst & $1(\mathrm{H})$ & $1(H)$ & 1.5 & & \\
\hline 8 & S. H. & 27 & $n$ & $\mathrm{Ph}$ & $1(H)$ & $1(+t)$ & & 1.0 & \\
\hline 9 & $\mathbf{M} \cdot \mathbf{T}$ & 30 & " & $\mathrm{He}$ & $1(H)$ & $1(H)$ & 0.2 & 0.4 & \\
\hline 10 & S. S. & 36 & m & $\mathrm{He}$ & $1(H)$ & $1(H)$ & 0.2 & 0.1 & \\
\hline 11 & H. S. & 24 & $\eta$ & $\mathrm{He}$ & $1(+t)$ & $1(H)$ & 0.35 & 0.4 & \\
\hline 12 & R. S. & 23 & $n$ & $\mathbf{H e}$ & $2(++)$ & $1(+4)$ & 0.8 & & \\
\hline 13 & S. A. & 24 & & $\mathrm{He}$ & $1(\mathrm{H})$ & $\mathbf{1}(H)$ & & 0.4 & \\
\hline 14 & M. M. & 23 & VII & D & $1(H)$ & $1(H)$ & & 0.6 & \\
\hline 15 & K. H. & 40 & $\eta$ & Br & $1(+H)$ & $1(\mathrm{WI})$ & & 0.5 & \\
\hline 16 & M.S. & 23 & " & D & $2(H)$ & $2(H)$ & 0.5 & & \\
\hline 17 & Y. T. & 26 & & D & $1(H)$ & $1(t)$ & & 0.6 & \\
\hline 18 & M. I. & 24 & VIII & An & $1(H)$ & $1(H)$ & 0.8 & 1.1 & \\
\hline 19 & T. W. & 24 & $n$ & G. I & $1(+4)$ & $1(H)$ & 1.1 & 1.2 & \\
\hline 20 & T. A. & 31 & $n$ & H. i.d & $1(H)$ & $1(H)$ & 1.2 & 0.9 & \\
\hline 21 & T. $\mathrm{K}$. & 20 & & D & $1(H)$ & $1(H)$ & 0.4 & & \\
\hline 22 & S. A. & 29 & VIIII & D & $1(\#)$ & $1(H)$ & & 0.7 & \\
\hline 23 & K. S. & 41 & $n$ & D & $1(H)$ & $1(\mathrm{H})$ & $\therefore$ & 0.8 & \\
\hline 24 & S. S. & 24 & 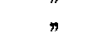 & Ep & $1(H)$ & $1(H)$ & 0.2 & & \\
\hline
\end{tabular}




\begin{tabular}{|c|c|c|c|c|c|c|c|c|c|}
\hline \multirow{2}{*}{$\begin{array}{l}\text { Case } \\
\text { No. }\end{array}$} & \multirow{2}{*}{ Name } & \multirow{2}{*}{$\begin{array}{l}\text { Years } \\
\text { of } \\
\text { age }\end{array}$} & \multirow{2}{*}{$\begin{array}{l}\text { Month } \\
\text { of } \\
\text { experi- } \\
\text { ment }\end{array}$} & \multirow{2}{*}{$\begin{array}{c}\text { Diagnosis? } \\
\text { of the } \\
\text { infants }\end{array}$} & \multicolumn{2}{|c|}{$\begin{array}{l}\text { Arakawa's } \\
\text { reaction }\end{array}$} & \multicolumn{2}{|c|}{$\begin{array}{c}\text { Glyoxalase } \\
\text { content }\end{array}$} & \multirow{2}{*}{ 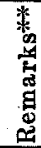 } \\
\hline & & & & & Right & Left & Right & Left & \\
\hline 25 & K. K. & 24 & VIII & G. I & $1(H)$ & $1(H)$ & 0.7 & & \\
\hline 26 & Y. U. & 27 & & D & $2(+1)$ & $2(H)$ & 1.0 & 1.2 & \\
\hline 27 & T. S. & 28 & IX & W & $1(H)$ & $1(H)$ & & 1.2 & \\
\hline 28 & K. K. & 33 & " & D & $1(H)$ & $1(H)$ & 0.35 & & \\
\hline 29 & M. I. & 21 & & $\mathrm{D}$ & $1(H)$ & $1(H)$ & 0.4 & & \\
\hline 30 & M. T. & 30 & & $\mathrm{D}$ & $1(H)$ & $1(H)$ & & 0.4 & $*$ \\
\hline 31 & K. I. & 25 & $\mathrm{X}$ & $\mathrm{D}$ & $1(\mathrm{H})$ & $1(\mathrm{HI})$ & 0.8 & 0.9 & \\
\hline 32 & I. A. & 29 & & $\mathbf{R}$ & $1($ (\#) & $1(\mathrm{H})$ & 1.2 & 0.8 & * \\
\hline 33 & M. F. & 22 & XI & $\mathrm{D}$ & $3(H)$ & $3(H)$ & 0.3 & 0.4 & \\
\hline 34 & M. K. & 41 & 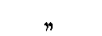 & W & $1(H)$ & $1(H)$ & & 1.0 & \\
\hline 35 & S. 0 . & 39 & & G. I & $1(+)$ & $3(+t)$ & 0.75 & 0.7 & \\
\hline 36 & М. T. & 30 & & Dyst & $2(H)$ & $1(H)$ & 0.7 & 0.9 & \\
\hline 37 & M. I. & 21 & XII & D & $1(H)$ & $1(H)$ & 0.5 & 1.0 & $\because$ \\
\hline 38 & S. M. & 29 & & D) & $1(+t)$ & $1(H)$ & 0.8 & 0.8 & \\
\hline 39 & M. T. & 26 & II & D & $5(H)$ & $5(H)$ & 0.2 & & \\
\hline 40 & S. S. & 27 & $n$ & D & $5(H)$ & $5(\mathrm{H})$ & & 0.3 & \\
\hline
\end{tabular}

**, $\S$ Cf. the note to Tables $1,2,3$ and 4 (p. 43).

Table of signs for showing different A rakawa's reaction.

$\begin{array}{lllll}1^{\prime} & 2^{\prime} & 3^{\prime} & 4^{\prime} & 5^{\prime}\end{array}$

1. 1 (\#) stands for $\mathrm{H}+\mathrm{H}$ H H H

2. $1(\mathrm{H})$ " $\mathrm{H}$.H $\mathrm{H}$

3. $2(\mathrm{H}), \quad+\mathrm{H}$ H H

4. $3(H) " H+H+H$

5. $4(\mathrm{H}), \quad+\mathrm{H}+\mathrm{H}$

6. $5(H) " H+H+H+H$

7. $1(+) \quad$ + $\#$ H H H

8. $2(+), \quad+$ H H H

9. $3(+) "+$ H + H

10. $4(+) "+H+H+$

11. $5(+) \quad$ + $\quad+$ H H H

12. $6(+) "+++H+H$

13. $7(+) "++H+H$

14. $8(+) "+++H+H$

15. $9(+) "+++H+H$

16. $10(+)$ " ++++ +

17. $11(+)$ +++++

18. $1( \pm) \quad$ $\quad+\mathrm{H} \mathrm{H} \mathrm{H}$

19. $2( \pm) \quad " \quad \pm+H+H$

20. $3( \pm), \quad \pm+H+H$

21. $4( \pm), \quad \pm++H$ H

22. $5( \pm)$,$++H$ H

23. 6(土) " $\pm+++H$

24. $7( \pm) \quad$ " $\quad \pm++++$ $\begin{array}{lllll}1^{\prime} & 2^{\prime} & 3^{\prime} & 4^{\prime} & 5^{\prime}\end{array}$ 25. $8( \pm)$ stands for $\pm \pm+\#$ \# 26. $9( \pm) \quad$ $\pm \pm+H+$ 27. 10 (士) $" \pm \pm++H$ 28. 11 (土) $" \quad \pm \pm+++$ 29. $12( \pm) \quad$ $\pm \pm \pm+H$ 30. $13( \pm) \quad " \quad \pm \pm \pm++$ 31. 14(土) $" \quad \pm \pm \pm \pm+$

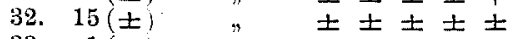
33. $1(-) \quad$ - $\pm+H$ H 34. $2(-) \quad$ - $\quad+$ H H 35. $3(-), \quad- \pm+++$ 36. $4(-), \quad- \pm+++$ 37. $5(-) \quad " \quad- \pm \pm++$ 38. 6(-) " $\quad- \pm \pm++$ 39. $7(-) \quad " \quad- \pm \pm \pm+$ 40. $8(-) \quad, \quad- \pm \pm \pm \pm$ 41. $9(-) \quad n-- \pm+H$ 42. $10(-) \quad, \quad-- \pm++$ 43. $11(-) \quad$ - \pm \pm+ 44. $12(-) \quad " \quad-- \pm \pm \pm$ 45. $13(-) \quad$ - -- \pm \pm 46. $14(-) \quad " \quad-- \pm \pm$ 47. $15(-) \quad " \quad-- \pm \pm$

48. $16(-), \quad,--- \pm$

Explanation to the table:-

Take, for instance, the sign: $2(H)$. This stands for A r a k a wa's reaction with the course (H) $1^{\prime}(H) 2^{\prime}(H) 3^{\prime}(H) 4^{\prime}(H) 5^{\prime}$. The sign does not express any prompt result of the reaction, so the prompt reaction of the sign: $2(H)$ may be $(-) 0^{\prime},( \pm) 0^{\prime}$, $(+) 0^{\prime}$ or even $(H) 0^{\prime}$, but this will not matter much, as the result of the reaction in one minute is the most important. 
Glyoxalase Content in Human Milk \& Ar a kawa's Reaction

TAble 2.

Glyoxalase content in the Arakawa-weakly positive group.

\begin{tabular}{|c|c|c|c|c|c|c|c|c|c|}
\hline \multirow{2}{*}{$\begin{array}{l}\text { Case } \\
\text { No. }\end{array}$} & \multirow{2}{*}{ Name } & \multirow{2}{*}{$\begin{array}{l}\text { Years } \\
\text { of } \\
\text { age }\end{array}$} & \multirow{2}{*}{$\begin{array}{l}\text { Month } \\
\text { of } \\
\text { experi- } \\
\text { ment }\end{array}$} & \multirow{2}{*}{$\begin{array}{c}\text { Diagnosis } \\
\text { of the } \\
\text { infants }\end{array}$} & \multicolumn{2}{|c|}{$\begin{array}{l}\text { Arakawa's } \\
\text { reaction }\end{array}$} & \multicolumn{2}{|c|}{$\begin{array}{c}\text { Glyoxalase } \\
\text { content }\end{array}$} & \multirow{2}{*}{ 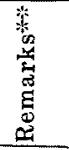 } \\
\hline & & & & & Right & Left & Right & Left & \\
\hline 1 & S. H. & 29 . & IV & Dyst & $14(-)$ & $1( \pm)$ & & 0.35 & $*$ \\
\hline 2 & F. A. & 26 & n. & I. $\mathrm{B}$ ? & $1(+)$ & $2(t)$ & & 0.2 & $*$ \\
\hline 3 & T. S. & 30 & & $\mathrm{He}$ & $1(H)$ & $15(-)$ & 0.4 & 0.3 & \\
\hline 4 & E. Y. & 32 & V & Pn & $16(-)$ & $5(-)$ & 0.4 & & \\
\hline 5 & M. A. & 25 & n & At & $1(-)$ & $15( \pm)$ & 0.5 & & $*$ \\
\hline 6 & M. A. & 29 & $n$ & D & $7( \pm)$ & $15( \pm)$ & 0.3 & & \\
\hline 7 & Y. 0. & 28 & $n$ & Ma & $16(-)$ & $4(+)$ & 1.3 & 1.5 & \\
\hline 8 & K. K. & 33 & $n$ & H. 0 & $8(+)$ & $8(+)$ & & 0.7 & \\
\hline 9 & K. 0 . & 30 & $n$ & D & $10(+)$ & $10(+)$ & 0.2 & 0.15 & \\
\hline 10 & M. S. & 29 & $n$ & $\mathrm{D}$ & $12( \pm)$ & $8(-)$ & 1.1 & 1.0 & \\
\hline 11 & K. I. & 28 & VI & Av. D & $11(-)$ & $10(-)$ & & 0.75 & \\
\hline 12 & A. A. & 25 & 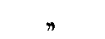 & $\mathbf{R}$ & $1(H)$ & $5( \pm)$ & 0.5 & 0.9 & \\
\hline 13 & M. M. & 30 & 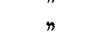 & $\operatorname{Pn}$ & $1(H)$ & $5( \pm)$ & 1.14 & 1.3 & \\
\hline 14 & H. K. & 41 & $\pi$ & D & $6(-)$ & $6( \pm)$ & 0.5 & & \\
\hline 15 & T. $\mathrm{G}$. & 22 & VII & L? & $1(+)$ & $1(+)$ & 0.4 & & \\
\hline 16 & M. T. & 31 & r & D & $10( \pm)$ & $4(-)$ & 0.3 & 0.4 & \\
\hline 17 & M. 0 . & 29 & $\pi$ & $W$ & $1(H)$ & $5(+)$ & 0.7 & 0.9 & \\
\hline 18 & M. S. & 23 & $n$ & D & $6( \pm)$ & $9(+)$ & 0.5 & & \\
\hline 19 & T. K. & 26 & 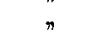 & $\mathbf{D}$ & $7(-)$ & $8(-)$ & & 1.0 & \\
\hline 20 & M. M. & 35 & 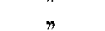 & D & $13( \pm)$ & $6(+)$ & & 0.23 & \\
\hline 21 & S. M. & 38 & $n$ & $\mathrm{Hy}$ & $13( \pm)$ & $13( \pm)$ & & 0.6 & \\
\hline 22 & C. S. & 29 & m & At & $6(二)$ & $16(-)$ & 1.2 & 1.0 & \\
\hline 23 & F. I. & 26 & $n$ & G. I & $6(+)$ & $11( \pm)$ & 0.4 & & \\
\hline 24 & s. s. & 30 & " & $\mathbf{R}$ & $7( \pm)$ & $5( \pm)$ & 0.3 & & \\
\hline 25 & M. S. & 29 & , & D & $1( \pm)$ & $1( \pm)$ & & 0.3 & \\
\hline 26 & K. I. & 28 & $\pi$ & D & $10(-)$ & $10(-)$ & 1.0 & & \\
\hline 27 & S. S. & 27 & 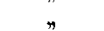 & D & $16(-)$ & $9(+)$ & 0.8 & & \\
\hline 28 & T. N. & 25 & , & H. i.d & $6(+)$ & $1(H)$ & 0.7 & 0.45 & \\
\hline 29 & M. 0 . & 22 & & $\mathrm{D}$ & $1(H)$ & $14(-)$ & 0.4 & 0.25 & \\
\hline 30 & K. $\mathrm{K}$. & 25 & VIII & $P_{n}$ & $12(-)$ & $3( \pm)$ & 0.3 & & \\
\hline 31 & T. M. & 28 & , & D) & $1(H)$ & $15( \pm)$ & 1.0 & 0.5 & \\
\hline 32 & M. T. & 29 & 》 & W & $16( \pm)$ & $4(+)$ & 0.4 & & \\
\hline 33 & H. K. & 28 & $n$ & H. 0 & $1(H)$ & $7( \pm)$ & 0.25 & 0.4 & \\
\hline 34 & R. Y. & 28 & $n$ & Ang & $6(-)$ & $1(H)$ & 0.3 & 0.4 & \\
\hline 35 & X. K. & 25 & 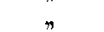 & $\mathrm{D}$ & $2( \pm)$ & $1(H)$ & 0.5 & 0.4 & \\
\hline 36 & H.S. & 32 & & H. MI & $1( \pm)$ & $1( \pm)$ & 0.7 & & \\
\hline 37 & T. K. & 34 & IX & D & $1(H)$ & 1 (士) & 0.2 & 0.25 & \\
\hline 38 & M. T. & 30 & 》 & Ic. $\mathrm{n}$ & $10( \pm)$ & $3(-)$ & 0.8 & & \\
\hline 39 & T. M. & 24 & $"$ & L? & $3(-)$ & $11(-)$ & & 0.5 & \\
\hline 40 & H. S. & 22 & ” & $\mathrm{Pn}$ & $13( \pm)$ & $7(+)$ & 0.3 & & \\
\hline 41 & K. T. & 23 & $n$ & $D$ & $16(-)$ & $7(-)$ & 0.25 & & $*$ \\
\hline 42 & K. T. & 32 & $\pi$ & Av & $3( \pm)$ & $1(H)$ & & 0.23 & * \\
\hline 43 & M. K. & 25 & " & An & $8(+)$ & $4(H)$ & 0.4 & & \\
\hline 44 & T. T. & 23 & , & $\mathrm{D}$ & $1(+4)$ & $5(+)$ & 0.45 & & \\
\hline 45 & T. N. & 28 & & D & $8( \pm)$ & $1(H)$ & 0.4 & 0.25 & \\
\hline 46 & C. $\mathrm{K}$. & 22 & $\mathrm{X}$ & I. $\mathrm{P}$ & $11( \pm)$ & $4(-)$ & 0.5 & & \\
\hline 47 & K. S. & 25 & , & G.I & $7( \pm)$ & $15(-)$ & 1.5 & & \\
\hline 48 & Y.S. & 23 & 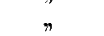 & Av. D & $2( \pm)$ & $2( \pm)$ & 1.7 & & $* \mathrm{Be}$ \\
\hline 49 & M. Y. & 19 & $"$ & D & $4(+)$ & $3(H)$ & 0.4 & & \\
\hline 50 & M. M. & 25 & $n$ & G. I & $16(-)$ & $1( \pm)$ & 0.2 & & \\
\hline 51 & M. I. & 32 & $\pi$ & $\mathbf{L}$ & $1(H)$ & $5(+)$ & 0.2 & 0.9 & \\
\hline 52 & T. S. & 21 & 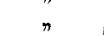 & W & $1(H)$ & $4(+)$ & 1.2 & 0.3 & \\
\hline
\end{tabular}




\begin{tabular}{|c|c|c|c|c|c|c|c|c|c|}
\hline \multirow{2}{*}{$\begin{array}{l}\text { Case } \\
\text { No. }\end{array}$} & \multirow{2}{*}{ Name } & \multirow{2}{*}{$\begin{array}{c}\text { Years } \\
\text { of } \\
\text { age }\end{array}$} & \multirow{2}{*}{$\begin{array}{l}\text { Month } \\
\text { of } \\
\text { experi- } \\
\text { ment }\end{array}$} & \multirow{2}{*}{$\begin{array}{c}\text { Diagnosis } \\
\text { of the } \\
\text { infants }\end{array}$} & \multicolumn{2}{|c|}{$\begin{array}{l}\text { Arakawa's } \\
\text { reaction }\end{array}$} & \multicolumn{2}{|c|}{$\begin{array}{c}\text { Glyoxalase } \\
\text { content }\end{array}$} & \multirow{2}{*}{ 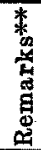 } \\
\hline & & & & & Right & Left & Right & Left & \\
\hline 53 & S. 0 . & 25 & $\mathrm{X}$ & D & $10(-)$ & $6( \pm)$ & 1.5 & & \\
\hline 54 & K. H. & 26 & $n$ & L? & $5( \pm)$ & $11( \pm)$ & 0.1 & 0.03 & \\
\hline 55 & I. $\mathrm{s}$. & 38 & $n$ & I. B & $1(+)$ & $1(+)$ & 0.25 & 0.2 & \\
\hline 56 & I. S. & 38 & 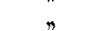 & $\mathrm{Br}$ & $11(-)$ & $3(-)$ & 0.15 & 0.4 & \\
\hline 57 & Y. M. & 20 & $n$ & H. M & $2(+)$ & $6(+)$ & 0.3 & 0.2 & \\
\hline 58 & A. W. & 29 & 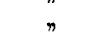 & Me.t & $4(-)$ & $4(-)$ & 0.1 & 0.1 & \\
\hline 59 & H. H. & 26 & $\eta$ & D & $7(+)$ & $3( \pm)$ & 0.2 & 0.25 & \\
\hline 60 & Y. Y. & 23 & " & $\mathrm{He}$ & $2(-)$ & $2(-)$ & 1.7 & 2.0 & \\
\hline 61 & K. S. & 28 & $n$ & Av? & $10(-)$ & $16(-)$ & 0.35 & 0.6 & \\
\hline 62 & E. M. & 26 & " & G. I & $6(-)$ & $16(-)$ & 0.7 & & \\
\hline 63 & K. H. & 27 & " & D & $10(-)$ & $14(-)$ & 1.2 & 0.7 & \\
\hline 64 & M. M. & 29 & $\eta$ & D & $11(-)$ & $3(-)$ & 1.5 & & \\
\hline 65 & Y. W. & 34 & " & I. P & $2( \pm)$ & $2( \pm)$ & 0.4 & 0.5 & $*$ \\
\hline 66 & F. K. & 38 & n & $A v$ & $1(H)$ & $5(+)$ & 0.1 & 0.15 & \\
\hline 67 & H. A. & 27 & $"$ & $\mathrm{Em}, \mathrm{s}$ & $7( \pm)$ & $8(-)$ & 1.7 & 1.2 & \\
\hline 68 & Y. M. & 30 & " & $\mathbf{R}$ & $1(+)$ & $1(+)$ & 0.25 & 0.3 & \\
\hline 69 & F. S. & 27 & $\pi$ & $A v$ & $7(-)$ & $14(-)$ & 0.3 & 0.25 & \\
\hline 70 & T. K. & 27 & $"$ & $\mathrm{Hg}$ & $11( \pm)$ & $9(+)$ & & 0.3 & \\
\hline 71 & S. A. & 25 & 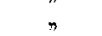 & G. I & $1(+)$ & $7(-)$ & 0.1 & 0.1 & \\
\hline 72 & K. I. & 28 & $\pi$ & Dyst & $11( \pm)$ & $11( \pm)$ & 0.6 & 0.8 & \\
\hline 73 & C. J. & 24 & $n$ & H. M & $4(+)$ & $4(+)$ & 0.6 & 0.6 & \\
\hline 74 & S. S. & 27 & 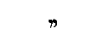 & D & $4(+)$ & $4(+)$ & 0.6 & & \\
\hline 75 & C. D. & 21 & $n$ & L? & $7(+)$ & $5( \pm)$ & 0.3 & 0.2 & \\
\hline 76 & K. I. & 28 & & D & $4( \pm)$ & 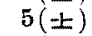 & 0.53 & & \\
\hline 77 & K. T. & 24 & XI & D & $7( \pm)$ & $14(-)$ & 0.4 & 0.3 & \\
\hline 78 & H. S. & 25 & n & I. $\mathrm{B}$ & $5( \pm)$ & $12(-6)$ & 0.2 & 0.5 & $*$ \\
\hline 79 & A. $\mathrm{K}$. & 24 & $"$ & $\mathrm{Ma}$ & $4(+)$ & $4(+)$ & 0.1 & 0.1 & \\
\hline 80 & K. S. & 28 & $n$ & D. $\mathrm{R}$ & $7(-)$ & $14(-)$ & 0.6 & 0.9 & \\
\hline 81 & M. M. & 31 & $"$ & $\mathbf{E}$ & $2(-)$ & $16(-)$ & 1.5 & & \\
\hline 82 & M. S. & 31 & $n$ & D & $1( \pm)$ & $2( \pm)$ & 0.4 & & $*$ \\
\hline 83 & S. M. & 29 & $\pi$ & D & $3( \pm)$ & $2( \pm)$ & 1.0 & 1.4 & \\
\hline 84 & K. A. & 21 & $"$ & $\mathrm{Ph}$ & $3( \pm)$ & $8( \pm)$ & 1.5 & & \\
\hline 85 & I. K. & 28 & n & $\mathrm{Br}$ & $2(+)$ & $2(+)$ & 0.8 & 0.8 & \\
\hline 86 & C. M. & 24 & $"$ & $\mathrm{~L}$ ? & $6(-)$ & $2( \pm)$ & 0.1 & 0.25 & \\
\hline 87 & E. S. & 25 & $n$ & $A v$ & 10 (士) & $5( \pm)$ & & 0.5 & \\
\hline 88 & T. Y. & 23 & $n$ & $A v$ & $16(-)$ & $5( \pm)$ & 0.1 & & \\
\hline 89 & M. K. & 28 & $n$ & $\mathrm{D}$ & $15(-)$ & $4(二)$ & & 0.5 & \\
\hline 90 & Y. S. & 23 & & G. I & $4( \pm)$ & $3(-)$ & 0.2 & & \\
\hline 91 & M. K. & 23 & XII & W & $1(-)$ & $7(+)$ & 0.2 & 0.4 & \\
\hline 92 & C.N. & 25 & $n$ & G. I & $15(-)$ & $4( \pm)$ & 0.3 & 0.2 & $*$ \\
\hline 93 & S. S. & 30 & $"$ & $\mathrm{D}$ & $1(H)$ & $3( \pm)$ & 1.0 & 1.5 & \\
\hline 94 & C. 0 & 39 & $n$ & $\mathrm{Br}$ & $6(-)$ & $3( \pm)$ & 0.6 & 1.0 & \\
\hline 95 & $\mathrm{M} . \mathrm{T}$ & 35 & $n$ & At & $1(+)$ & $1( \pm)$ & & 0.3 & \\
\hline 96 & H. S. & 24 & $"$ & G. I & $12( \pm)$ & $9( \pm)$ & 0.2 & 0.15 & \\
\hline 97 & H. H. & 32 & $"$ & D & $13( \pm)$ & $12(-)$ & 0.05 & 0.2 & \\
\hline 98 & S. K. & 25 & $"$ & Hyd & $10(-)$ & $10(-)$ & 0.3 & 0.2 & * \\
\hline 99 & I. $\mathbf{S}$. & 23 & $n$ & $\mathrm{D}$ & $7( \pm)$ & $16(-)$ & 1.2 & 1.5 & $*$ \\
\hline 100 & E. K. & 24 & I & G. I & $6(-)$ & $4(+)$ & 0.1 & 0.2 & \\
\hline 101 & M.T. & 22 & n & $\mathrm{He}$ & $6(-)$ & $16(-)$ & 0.2 & 0.3 & \\
\hline 102 & E. K. & 27 & $n$ & Av. D & $16(-)$ & $11(-)$ & 0.4 & 0.3 & \\
\hline 103 & H. N. & 37 & $"$ & G. I & $11( \pm)$ & $5( \pm)$ & 0.25 & 0.3 & \\
\hline 104 & T. C. & 25 & & Av. D & $8(-)$ & $15(二)$ & & 0.2 & $*$ \\
\hline 105 & T. G. & 38 & II & At & $16(-)$ & $6(-)$ & 0.3 & & \\
\hline 106 & H. I. & 26 & $\pi$ & $\mathbf{R}$ & $4(+)$ & $9(+)$ & 0.5 & & \\
\hline 107 & S. IK. & 29 & $n$ & G. I & $5(+)$ & $4(+)$ & 0.3 & 0.2 & * \\
\hline 108 & C. W. & 33 & $n$ & D & $1(H)$ & $6( \pm)$ & 0.4 & 0.5 & \\
\hline
\end{tabular}


Glyoxalase Content in Human Milk \& A raka wa's Reaction

\begin{tabular}{|c|c|c|c|c|c|c|c|c|c|}
\hline \multirow{2}{*}{$\begin{array}{l}\text { Case } \\
\text { No. }\end{array}$} & \multirow{2}{*}{ Name } & \multirow{2}{*}{$\begin{array}{c}\text { Years } \\
\text { of } \\
\text { age }\end{array}$} & \multirow{2}{*}{$\begin{array}{l}\text { Month } \\
\text { of } \\
\text { experi- } \\
\text { ment }\end{array}$} & \multirow{2}{*}{ 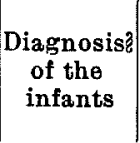 } & \multicolumn{2}{|c|}{$\begin{array}{l}\text { Arakawa's } \\
\text { reaction }\end{array}$} & \multicolumn{2}{|c|}{$\begin{array}{c}\text { Glyoxalase } \\
\text { content }\end{array}$} & \multirow{2}{*}{ 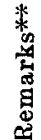 } \\
\hline & & & & & Right & Left & Right & Left & \\
\hline 109 & S. H. & 40 & II & Av & $14( \pm)$ & $8(-)$ & 0.1 & 0.1 & \\
\hline 110 & K. S. & 27 & III & D & $4(+)$ & $4(+)$ & 0.2 & & \\
\hline 111 & Y. T. & 32 & n & $\mathrm{He}$ & $8(-)$ & $15(-)$ & & 0.2 & \\
\hline 112 & K. S. & 26 & $\pi$ & Em. $\mathbf{s}$ & $6(-)$ & $6(-)$ & 0.07 & 0.1 & \\
\hline 113 & T. 0. & 31 & $n$ & $\mathbf{R}$ & $2(H)$ & $16(-)$ & 0.25 & 0.4 & \\
\hline 114 & T. S. & 25 & $n$ & Dyst & $4(+)$ & $4(+)$ & 0.1 & 0.1 & \\
\hline 115 & S. S. & 25 & $"$ & $\mathbf{E}$ & $8(-)$ & $8(-)$ & & 0.8 & \\
\hline 116 & K. K. & 27 & $n$ & $\mathrm{Py}$ & $\mathbf{3}(-)$ & $3(-)$ & 0.1 & 0.2 & \\
\hline
\end{tabular}

§, ** Cf. the note to Tables $1,2,3$ and 4 (p. 43).

TAble 3.

Glyoxalase content in the Arakawa-negative group.

\begin{tabular}{|c|c|c|c|c|c|c|c|c|c|}
\hline \multirow{2}{*}{$\begin{array}{l}\text { Case } \\
\text { No. }\end{array}$} & \multirow{2}{*}{ Name } & \multirow{2}{*}{$\begin{array}{l}\text { Years } \\
\text { of } \\
\text { age }\end{array}$} & \multirow{2}{*}{$\begin{array}{c}\text { Month } \\
\text { of } \\
\text { experi- } \\
\text { ment }\end{array}$} & \multirow{2}{*}{$\begin{array}{c}\text { Diagnosis } \\
\text { of the } \\
\text { infants }\end{array}$} & \multicolumn{2}{|c|}{$\begin{array}{c}\text { Arakawa's } \\
\text { reaction }\end{array}$} & \multicolumn{2}{|c|}{$\begin{array}{c}\text { Glyoxalase } \\
\text { content }\end{array}$} & \multirow{2}{*}{ 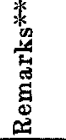 } \\
\hline & & & & & Right & Left & Right & Left & \\
\hline 1 & K. K. & 32 & IV & Ic. $\mathbf{n}$ & $16(-)$ & $16(-)$ & 1.55 & & $*$ \\
\hline 2 & K. K. & 22 & & At & $16(-)$ & $16(-)$ & 0.8 & 0.7 & $*$ \\
\hline 3 & T. K. & 30 & $\dot{V}$ & $\mathrm{D}$ & $16(-)$ & $16(-)$ & 0.5 & 0.3 & * \\
\hline 4 & T. S. & 28 & $n$ & G. I & $16(-)$ & $16(-)$ & 1.2 & & $\because \mathrm{Be}$ \\
\hline 5 & Y. 0 . & 22 & $n$ & I. B ? & $14(-)$ & $16(-)$ & 0.6 & 0.9 & \\
\hline 6 & S. 0 . & 27 & $n$ & D & $14(-)$ & $16(-)$ & 0.6 & 0.35 & \\
\hline 7 & K. S. & 28 & $m$ & L & $14(-)$ & $16(-)$ & 0.2 & 0.4 & \\
\hline 8 & I. $\mathrm{S}$. & 30 & VI & D & $16(-)$ & $16(-)$ & 0.45 & 0.6 & \\
\hline 9 & M.S. & 34 & VII & Dyst & $16(-)$ & $16(-)$ & & 1.0 & \\
\hline 10 & M. M. & 24 & 》 & D & $16(-)$ & $16(-)$ & 1.0 & & 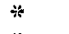 \\
\hline 11 & T. N. & 25 & 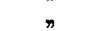 & $P_{n}$ & $15(-)$ & $15(-)$ & 0.8 & & $*$ \\
\hline 12 & T. A. & 32 & VIII & Pn & $16(-)$ & $16(-)$ & & 0.6 & \\
\hline 13 & W.T. & 24 & IX & D & $16(-)$ & $14(-)$ & & 0.5 & \\
\hline 14 & K. H. & 25 & " & I. P ? & $15(-)$ & $15(-)$ & 1.0 & & $* \mathrm{Be}$ \\
\hline 15 & K. I. & 26 & " & $\mathbf{R}$ & $16(-)$ & $16(-)$ & 0.5 & 0.6 & $*$ \\
\hline 16 & K. 0. & 23 & 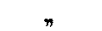 & D & $15(-)$ & $15(-)$ & 0.1 & 0.2 & \\
\hline 17 & S. M. & 22 & $\mathbf{x}$ & D & $16(-)$ & $16(-)$ & 0.4 & & \\
\hline 18 & F. H. & 31 & $n$ & D & $16(-)$ & $16(-)$ & 0.5 & & \\
\hline 19 & K. A. & 26 & n & H.M & $16(-)$ & $16(-)$ & 0.2 & 0.2 & \\
\hline 20 & T. K. & 21 & " & D & $14(-)$ & $16(-)$ & 0.4 & 0.7 & \\
\hline 21 & S. M. & 27 & n & D & $16(-)$ & $16(-)$ & 0.4 & 0.3 & $*$ \\
\hline 22 & S. E. & 27 & $\pi$ & Av. D & $15(-)$ & $15(-)$ & 0.5 & 0.5 & \\
\hline 23 & T. T. & 21 & $n$ & At & $15(-)$ & $16(-)$ & 0.25 & 0.2 & $*$ \\
\hline 24 & N. S. & 31 & $\pi$ & D & $15(-)$ & $16(-)$ & 0.3 & 0.1 & $*$ \\
\hline 25 & F. 0 . & 23 & 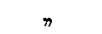 & D & $16(-)$ & $16(-)$ & 0.2 & 0.2 & \\
\hline 26 & R. D. & 25 & & G. I & $16(-)$ & $16(-)$ & 0.1 & 0.15 & $*$ \\
\hline 27 & K. 0 . & 34 & XI & $\mathbf{R a}$ ? & $16(-)$ & $16(-)$ & 0.2 & 0.5 & \\
\hline 28 & C. 0 . & 23 & n & D & $16(-)$ & $16(-)$ & 0.4 & 0.2 & \\
\hline 29 & S. K. & 40 & n & Av & $16(-)$ & $16(-)$ & 0.3 & 0.2 & \\
\hline 30 & T. T. & 26 & " & D & $16(-)$ & $16(-)$ & 0.1 & 0.1 & \\
\hline 31 & S. M. & 21 & ” & $\mathbf{A v}$ & $16(-)$ & $14(-)$ & 0.1 & 0.03 & \\
\hline 32 & K. A. & 20 & " & D & $15(-)$ & $15(-)$ & 0.1 & 0.2 & \\
\hline 33 & A. M. & 25 & n & I. B? & $16(-)$ & $16(-)$ & 0.6 & 0.7 & * \\
\hline
\end{tabular}




\begin{tabular}{|c|c|c|c|c|c|c|c|c|c|}
\hline \multirow{2}{*}{$\begin{array}{l}\text { Case } \\
\text { No. }\end{array}$} & \multirow{2}{*}{ Name } & \multirow{2}{*}{$\begin{array}{c}\text { Years } \\
\text { of } \\
\text { age }\end{array}$} & \multirow{2}{*}{$\begin{array}{l}\text { Month } \\
\text { of } \\
\text { experi- } \\
\text { ment }\end{array}$} & \multirow{2}{*}{$\begin{array}{c}\text { Diagnosis } \\
\text { of the } \\
\text { infants }\end{array}$} & \multicolumn{2}{|c|}{$\begin{array}{c}\text { Arakawa's } \\
\text { reaction }\end{array}$} & \multicolumn{2}{|c|}{$\begin{array}{c}\text { Glyoxalase } \\
\text { content }\end{array}$} & \multirow{2}{*}{ 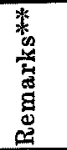 } \\
\hline & & & & & Right & Left & Right & Left & \\
\hline 34 & S. K. & 41 & XI & $L$ ? & $16(-)$ & $14(-)$ & 0.03 & 0.05 & \\
\hline 35 & M. M. & 25 & 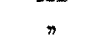 & $\mathbf{P n}_{n}$ & $16(-)$ & $16(-)$ & 1.6 & & \\
\hline 36 & M. S. & 28 & $\pi$ & At & $16(-)$ & $16(-)$ & & 0.05 & \\
\hline 37 & A. H. & 28 & XII & L? & $16(-)$ & $16(-)$ & 0.05 & 0.05 & \\
\hline 38 & I. 0 . & 31 & $n$ & Fm.s & $16(-)$ & $16(-)$ & 0.2 & 0.4 & \\
\hline 39 & M. 0 . & 34 & $n$ & $\mathbf{R}$ & $16(-)$ & $16(-)$ & 0.3 & 0.25 & \\
\hline 40 & K. A. & 20 & $n$ & Dyst & $15(-)$ & $15(-)$ & 1.2 & 0.8 & $*$ \\
\hline 41 & M. N. & 26 & 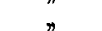 & D & $16(-)$ & $16-$ & 0.5 & 0.6 & * \\
\hline 42 & K. U. & 27 & $n$ & H. i. d & 15 & $15(-)$ & 0.6 & 0.5 & \\
\hline 43 & S. T. & 23 & $n$ & L & $16(-)$ & $16(-)$ & 1.1 & 0.8 & \\
\hline 44 & M. S. & 21 & $n$ & $\overline{\mathrm{L}}$ ? & $15(-)$ & $16(-)$ & 0.4 & 0.45 & \\
\hline 45 & I. $\mathrm{S}$. & 27 & $n$ & $\mathrm{D}$ & $16(-)$ & $16(-)$ & 0.1 & 0.1 & \\
\hline 46 & S. H. & 41 & $n$ & $\mathrm{Br}$ & $15(-)$ & $16(-)$ & 0.4 & 0.3 & \\
\hline 47 & Y. O. & 26 & 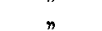 & $A v$ & $16(-)$ & $16(-)$ & 0.2 & 0.2 & $* \mathrm{Be}$ \\
\hline 48 & W.K. & 24 & $r$ & $\mathrm{Br}$ & $16(-)$ & $16(-)$ & 0.1 & 0.15 & \\
\hline 49 & T. F. & 25 & & $\mathrm{Br}$ & $16(-)$ & $16(-)$ & & 1.4 & \\
\hline 50 & S. Y. & 26 & $\mathrm{I}$ & $\mathrm{D}$ & $16(-)$ & $15(-)$ & & 0.3 & \\
\hline 51 & A. $\mathbf{H}$. & 26 & $n$ & $\mathrm{Hg}$ & $16(-)$ & $16(-)$ & & 0.3 & \\
\hline 52 & K. S. & 29 & 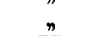 & $\mathrm{Br}$ & $15(-)$ & $16(-)$ & 0.35 & & \\
\hline 53 & K. U. & 30 & II & At & $16(-)$ & $16(-)$ & 0.2 & 0.1 & \\
\hline 54 & H. S. & 27 & III & I. $B$ & $16(-)$ & $16(-)$ & 0.05 & 0.1 & \\
\hline 55 & N. T. & 24 & " & At & $16(-)$ & $16(-)$ & 0.2 & & \\
\hline 56 & T. N. & 23 & $n$ & At & $16(-)$ & $16(-)$ & 0.3 & & \\
\hline
\end{tabular}

$\S$, * Cf. the note to Tables $1,2,3$ and 4 (p. 43).

left side, and in the weakly Arakawa-positive group it was 0.53 on the right side and 0.50 on the left. In the Arakawa-negative group it was 0.45 on the right and 0.39 on the left (Cf. Table 6).

As has been shown in Table 4 and Table 7, I estimated in 23 cases with different Arakawa's reaction on both breasts, and found the average content on the side with milk with $1^{\prime}(+)$ side to be 0.54 and on the other side to be 0.52 (Cf. Table 7).

As has been shown in Table 8, the glyoxalase content was found in the highest per cent (42.4\%) in the Arakawa-positive group in 0.99 $\sim 0.5$ group, in the weakly Arakawa-positive group it was found in the highest per cent (59.2\%) in 0.49 0.1 group, and in the completely Arakawa-negative group it was found in the highest per cent $(56.4 \%)$ in $0.49 \sim 0.1$ group. Now, if all the cases were divided according to the glyoxalase content into subgroups-Subgroup I with more than 2.0, Subgroup II with 1.99 1.0, Subgroup III with 0.99 0.5, Subgroup IV with $0.49 \sim 0.1$, Subgroup V with less than 0.09, then the distribution of all the cases belonging to the three groups according to the intensity of Arakawa's reaction will be as is given in Table 8 . 
Table 4.

Glyoxalase content of mothers with different Arakawa's reaction on both breasts.

\begin{tabular}{|c|c|c|c|c|c|c|c|c|c|}
\hline \multirow{2}{*}{$\begin{array}{l}\text { Case } \\
\text { No. }\end{array}$} & \multirow{2}{*}{ Name } & \multirow{2}{*}{$\begin{array}{c}\text { Years } \\
\text { of } \\
\text { age }\end{array}$} & \multirow{2}{*}{$\begin{array}{l}\text { Month } \\
\text { of } \\
\text { experi- } \\
\text { ment }\end{array}$} & \multirow{2}{*}{$\begin{array}{c}\text { Diagnosis } \\
\text { of the } \\
\text { infants }\end{array}$} & \multicolumn{2}{|c|}{$\begin{array}{l}\text { Arakawa's } \\
\text { reaction }\end{array}$} & \multicolumn{2}{|c|}{$\begin{array}{c}\text { Glyoxalase } \\
\text { content }\end{array}$} & \multirow{2}{*}{ 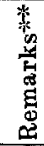 } \\
\hline & & & & & Right & Left & Right & Left & \\
\hline 1 & T. S. & 30 & IV & $\mathrm{He}$ & $1(H)$ & $15(-)$ & 0.4 & 0.3 & \\
\hline 2 & R. 0 . & 30 & & $\mathrm{He}$ & $1(++)$ & $16(-)$ & 0.9 & 0.1 & \\
\hline 3 & S. W. & 28 & & $\mathrm{He}$ & $15(-)$ & $1(H)$ & 0.2 & 0.5 & \\
\hline 4 & A. A. & 25 & VI & $\mathrm{R}$ & $1(H)$ & $5( \pm)$ & 0.5 & 0.9 & \\
\hline 5 & M. M. & 30 & & Pn & $1(H)$ & $6( \pm)$ & 1.14 & 1.3 & \\
\hline 6 & M. 0 . & 29 & VII & W & $1($ \#) & $8(+)$ & 0.7 & 0.9 & \\
\hline 7 & T. N. & 25 & $"$ & H.i.d & $7(+)$ & $1(H)$ & 0.7 & 0.45 & \\
\hline 8 & M. 0 . & 22 & & D & $1(H)$ & $14(-)$ & 0.4 & 0.25 & \\
\hline 9 & T. M. & 28 & VIII & D & $1(+)$ & $15( \pm)$ & 1.0 & 0.5 & \\
\hline 10 & H. K. & 28 & $\pi$ & H. O & $1(H)$ & $7( \pm)$ & 0.25 & 0.4 & \\
\hline 11 & Y. K. & 25 & 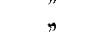 & D & $3( \pm)$ & $1(\bar{H})$ & 0.5 & 0.4 & \\
\hline 12 & R. Y. & 28 & & Ang & $6(-)$ & $1(H)$ & 0.3 & 0.4 & \\
\hline 13 & T. K. & 34 & IX & D & $1(4)$ & $1( \pm)$ & 0.2 & 0.25 & \\
\hline 14 & T. N. & 28 & 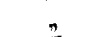 & D & $8( \pm)$ & $1(H)$ & 0.4 & 0.25 & \\
\hline 15 & M. I. & 32 & $\mathrm{X}$ & $\mathrm{L}$ & $1(H)$ & $5(+)$ & 0.2 & 0.9 & \\
\hline 16 & T. $\mathrm{s}$. & 21 & " & W & $1(H)$ & $5(+)$ & 1.2 & 0.3 & \\
\hline 17 & F. K. & 38 & $"$ & $\mathrm{Av}$ & $1(t+)$ & $5(+)$ & 0.1 & 0.15 & \\
\hline 18 & K. W. & 29 & $n$ & Me.t & $1(+)$ & $16(-)$ & 0.2 & 0.2 & \\
\hline 19 & S. M. & 29 & XI & D & $3( \pm)$ & $1(+)$ & 1.0 & 1.4 & \\
\hline 20 & S. S. & 30 & XII & D & $1(H)$ & $3( \pm)$ & 1.0 & 1.5 & $\%$ \\
\hline 21 & I. S. & 33 & , & $\mathrm{Br}$ & $16(-)$ & $1(t+)$ & 0.1 & 0.2 & $\ddot{*}$ \\
\hline 22 & C. W. & 33 & II & D & $1(H)$ & $10(-)$ & 0.4 & 0.5 & $*$ \\
\hline 23 & T. 0 . & 31 & III & $\mathbf{R}$ & $2(H)$ & $16(-)$ & 0.25 & 0.4 & \\
\hline
\end{tabular}

$\S$, $*$ Cf. the note to Tables $1,2,3$ and 4 (see below).

Note to Tables 1, 2, 3 and 4 .

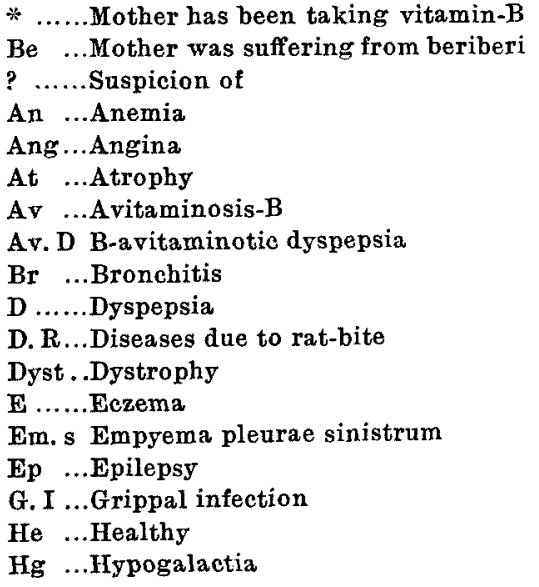

* .......Mother has been taking vitamin-B

Be Mother was suffering from beriberi

An ...Anemia

Ang...Angina

Av ...Avitaminosis-B

Av. D B-avitaminotic dyspepsia

D. R...Diseases due to rat-bite

Dyst...Dystrophy

Em. s Empyema pleurae sinistrum

He ...Healthy

H. i. d Hernia inguinalis dextra

H. M...Heine-Medin's disease

H. 0 ...Habitual obstipation

Hy ...Hydrocephalus

Hyd ...Hydrocele

I. B ...Infantile beriberi

I. P ...Infantile preberiberi

Ic. n...Icterus neonatorum

L ......Lues congenita

Ma ...Malnutrition

Me. t...Meningitis tuberculosa

$\mathrm{Ph}$...Pharyngitis

Pn ...Pneumonia

Py ...Pylorospasm

$\mathrm{R}$......Rhinopharyngitis

Ra ...Rachitis

W ...Whooping cough 
TABLE 5. Arakawa's reaction and glyoxalase content in human milk.

\begin{tabular}{|c|c|c|c|}
\hline $\begin{array}{l}\text { Arakaw a's } \\
\text { reaction }\end{array}$ & $\begin{array}{l}\text { Cases } \\
\text { in all }\end{array}$ & $\begin{array}{c}\text { Cases with more } \\
\text { than } 1.0 \\
(\%)\end{array}$ & $\begin{array}{c}\text { Glyoxalase a verage } \\
\text { content }\end{array}$ \\
\hline $\begin{array}{l}\text { Positive group } \\
\text { Weakly positive group } \\
\text { Negative group }\end{array}$ & $\begin{array}{r}59 \\
179 \\
94\end{array}$ & $\begin{array}{rr}16 & (27.1 \%) \\
30 & (16.8 \% \%) \\
9 & (9.6 \%)\end{array}$ & $\begin{array}{l}0.73 \\
0.52 \\
0.42\end{array}$ \\
\hline Total & 332 & $55 \quad(16.7 \% 0)$ & 0.53 \\
\hline
\end{tabular}

Table 6.

Arakawa's reaction and glyoxalase content on both breasts.

\begin{tabular}{|c|c|c|c|c|}
\hline $\begin{array}{l}\text { Arakawa's } \\
\text { reaction }\end{array}$ & \multicolumn{2}{|c|}{ Cases in all $\left\{\begin{array}{l}R=\text { right } \\
L=\text { left }\end{array}\right.$} & \multicolumn{2}{|c|}{$\begin{array}{c}\text { Glyoxalase average } \\
\text { content }\end{array}$} \\
\hline Positive group & $59\left\{\begin{array}{l}\mathrm{R} \\
\mathrm{L}\end{array}\right.$ & $\begin{array}{l}29 \\
30\end{array}$ & $\begin{array}{l}\mathbf{R} \\
\mathbf{L}\end{array}$ & $\begin{array}{l}0.72 \\
0.74\end{array}$ \\
\hline Weakly positive group & $179\left\{\begin{array}{l}\mathrm{R} \\
\mathrm{L}\end{array}\right.$ & $\begin{array}{l}99 \\
80\end{array}$ & $\begin{array}{l}\mathbf{R} \\
\mathbf{L}\end{array}$ & $\begin{array}{l}0.53 \\
0.50\end{array}$ \\
\hline Negative gronp & $94\left\{\begin{array}{l}\mathbf{R} \\
\mathbf{L}\end{array}\right.$ & $\begin{array}{l}49 \\
45\end{array}$ & $\begin{array}{l}\mathbf{R} \\
\mathrm{L}\end{array}$ & $\begin{array}{l}0.45 \\
0.39\end{array}$ \\
\hline & TABLE & & & \\
\hline
\end{tabular}

Glyoxalasc content in human milk with different Arakawa's reaction on both breasts.

\begin{tabular}{|c|c|c|}
\hline \multicolumn{2}{|c|}{ Cases in all $\begin{array}{c}\text { Side with Arakaw a's reaction } 1^{\prime}(\#) \\
\text { The other side with much weaker } \\
\text { Arakawa's reaction }\end{array}$} & Glyoxalase average content \\
\hline 46 & $\begin{array}{l}23 \\
23\end{array}$ & $0.53\left\{\begin{array}{l}\text { Side with Arak a w a's } \\
\text { reaction } 1^{\prime}(H) \quad 0.54 \\
\text { The other side with } \\
\text { much weaker Ara- } \\
\text { kawa's reaction } 0.52\end{array}\right.$ \\
\hline
\end{tabular}

If all the cases are distributed according to the seasons of the year, then the average content of the glyoxalase will be as shown in Table 9 . In the Arakawa-positive group it was 0.72 in spring, 0.79 in summer, 0.72 in autumn and 0.60 in winter. In the weakly Arakawa-positive group it was 0.44 in spring, 0.59 in summer, 0.55 in autumn and 0.41 in winter, and in the completely Arakawa-negative group it was 0.55 in spring, 0.74 in summer, 0.33 in autumn and 0.41 in winter. Of all $\mathrm{my}$ 
Table 8.

Distribution of the cases according to the intensity of A rakawa's reaction and the content of glyoxalase.

\begin{tabular}{|c|c|c|c|c|c|c|}
\hline Arakawa's rcaction & $\begin{array}{l}7 \\
7 \\
.7 \\
0 \\
0 \\
0 \\
0 \\
0 \\
0\end{array}$ & 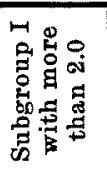 & 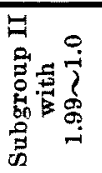 & 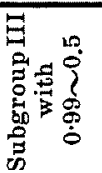 & 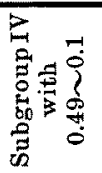 & 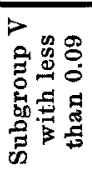 \\
\hline Arakawa-positive group & 59 & $\begin{array}{r}1.7 \% \\
(1)^{*}\end{array}$ & $\begin{array}{c}25.4 \% \\
(15)\end{array}$ & $\begin{array}{c}42.4 \% \\
(25)\end{array}$ & $\begin{array}{c}30.5 \% \\
(18)\end{array}$ & ${ }^{0}$ \\
\hline Arakawa-weakly positive group & 179 & $\begin{array}{c}0.5 \% \\
(1)\end{array}$ & $\begin{array}{c}16.2 \% \\
(29)\end{array}$ & $\begin{array}{c}22.4 \% \\
(40)\end{array}$ & $\begin{array}{r}59.2 \% \\
(106)\end{array}$ & $\begin{array}{c}1.7 \% \\
(3)\end{array}$ \\
\hline Arakawa-negative group & 94 & ${ }^{0}(0)$ & $\begin{array}{l}9.6 \% \\
(9)\end{array}$ & $\begin{array}{c}26.6 \% \\
(25)\end{array}$ & $\begin{array}{c}56.4 \% \\
(53)\end{array}$ & $\begin{array}{c}7.4 \% \\
(7)\end{array}$ \\
\hline
\end{tabular}

* Number of cases.

materials, it was 0.56 in spring, 0.67 in summer, 0.50 in autumn and 0.43 in winter.

TABLE 9.

Seasonal variation of glyoxalase content in human milk.

\begin{tabular}{l|l|l|l|l}
\hline Arak aw a's reaction & Spring & Summer & Autumn & Winter \\
\hline Positive group & & & & \\
Weakly positive group & $0.74(21)^{*}$ & $0.79(17)$ & $0.72(15)$ & $0.60(6)$ \\
Negative group & $0.55(16)$ & $0.74(6)$ & $0.33(42)$ & $0.41(30)$ \\
\hline Total & $0.56(62)$ & $0.67(60)$ & $0.50(140)$ & $0.43(70)$ \\
\hline
\end{tabular}

* Number of cases.

Table 10 will show the relation between the age of mothers and the average content of glyoxalase; in the group of mothers of 19 25 years of age the content of glyoxalase was 0.55 , in the group of mothers of $26 \sim 30$ years of age, 0.59 , in the group of mothers of $31 \sim 35$ years of age, 0.52 and in the group of mothers of $36 \sim 41$ years of age, 0.41 .

Table 10.

Relation between the glyoxalase content and the years of age of mothers.

\begin{tabular}{c|c|c}
\hline Years of age of mothers & Cases in all & Glyoxalase average content \\
\hline $19-25$ & 83 & 0.55 \\
$26-30$ & 86 & 0.59 \\
$31-35$ & 26 & 0.52 \\
$36-41$ & 17 & 0.41
\end{tabular}


As to the distribution of the content of glyoxalase according to the diseases of the infants, it was, as will be seen in Table 11, 0.19 in the case of empyema pleurae, 0.23 in avitaminosis- $\mathrm{B}, 0.30$ in hypogalactia, 0.34 in lues congenita, 0.37 in infantile beriberi, 0.39 in atrophy, 0.81 in eczema, 1.18 in icterus neonatorum and 1.25 in pharyngitis. The number of cases of each disease is scanty, so that of course no definite conclusion can be drawn from the table. But from the result shown in this table it will be instructive to see the result in a much larger number of materials.

TABle 11.

Relation between the glyoxalase content in mothers' milk and the diseases of infants.

\begin{tabular}{l|r|r||l|l|l}
\hline Diagnosis of infants & Cases & $\begin{array}{c}\text { Glyoxalase } \\
\text { average } \\
\text { content }\end{array}$ & Diagnosis of infants & Cases & $\begin{array}{c}\text { Glyoxalase } \\
\text { average } \\
\text { content }\end{array}$ \\
\hline Empyema pleurae & 2 & 0.19 & Healthy & 10 & 0.61 \\
Avitaminosis-B & 10 & 0.23 & Infantile preberiberi & 3 & 0.63 \\
Hypogalactia & 2 & 0.30 & Anemia & 2 & 0.68 \\
Lues congenita & 11 & 0.34 & Hernia & 3 & 0.71 \\
Infantile beriberi & 6 & 0.37 & Pneumonia & 7 & 0.74 \\
Atrophy & 11 & 0.39 & Whooping cough & 6 & 0.75 \\
Heine-Medin's disease & 4 & 0.44 & Malnutrition & 2 & 0.75 \\
Rhinopharyngitis & 8 & 0.49 & Dystrophy & 7 & 0.78 \\
Grippal infection & 17 & 0.51 & Eczema & 4 & 0.81 \\
Obstipation & 2 & 0.52 & Icterus neonatorum & 2 & 1.18 \\
Dyspepsia & 75 & 0.57 & Pharyngitis & 2 & 1.25 \\
Bronchitis & 8 & 0.57 & &
\end{tabular}

$\S$ It is to be noted that the diagnosis refers to infants and that glyoxalase content refers to mothers.

\section{Comment.}

As has been shown in Table 5 , the glyoxalase content was identified in a much larger amount in the milk in the Arakawa-positive case. As has been shown in Table 6 the glyoxalase content was identified in a larger amount in the right and left in the Arakawa-positive case. In the case of milk with different Arakaw a's reaction on both breasts, I did not find a remarkable difference between them (Cf. Table 7). From this result it seems that milk of mothers with Arakawa's reaction positive only on one side and with the very weak reaction on the other may not be a good one, if the absolute glyoxalase content is taken into consideration. Table 8 will show very clearly that the Arakawapositive group is rich in glyoxalase content. The glyoxalase content 
was the largest in summer and the smallest in winter, and it was the largest in the group of mothers of $26 \sim 30$ years of age and the smallest in the group of mothers of $36 \sim 41$ years of age. The difference of glyoxalase content according to four seasons or according to mothers' years of age will be by far smaller as compared with that according to the intensity of Arakawa's reaction.

As has been seen, glyoxalase content of human milk is different most distinctly according to the intensity of Arakawa's reaction. Arakawa-positive milk is richer in the enzyme than Arakawa-negative milk. Now Taka matsu ${ }^{13)}$ was able to identify methyl glyoxal-like substance in Arakawa-negative milk. If his result and mine are combined, the relation will be interesting, because the methyl glyoxal-like substance is found in Arakawa-negative milk in which glyoxalase is present in a small amount.

It is further interesting to take into consideration Findlay's?) report that the glyoxalase content of the liver in pigeons with beriberi is less than that in control pigeons, and that an administration of vitamin-B to a beriberic pigeon is followed by an increase in the glyoxalase content of the liver, especially because an administration of vitamin-B to such pigeons will, according to $\mathrm{Findlay},{ }^{7)}$ increase the glyoxalase content of the liver and because Arakawa-negative milk will, as Takamats u's ${ }^{14)}$ and my experience will show, become richer in glyoxalase, on an administration of vitamin B to mothers secreting such a milk. That experience of mine is published in the next paper. ${ }^{15}$ It will then be highly probable that human milk negative to Ara$\mathrm{kaw}$ a's reaction is a milk from B-avitaminotic body.

\section{Conclusion.}

Human milk positive to Arakawa's reaction is rich in glyoxalase, while Arakawa-negative milk is poor in it. This is interesting in consideration of Takamatsu's ${ }^{13)}$ result that methyl glyoxal-like substance is found in Arakawa-negative milk.

A mother with different Arakawa's reaction on both breasts seems to secret milk of an almost equal glyoxalase content, and then the content is not large.

15) R. Orim o, Tohoku J. Exp. Med., 1938, 35, 48. 